\title{
Diseño y Validación de la Escala de Sensibilidad al Rechazo Amoroso (SRA)
}

\section{Design and Validation of the Loving Rejection Sensitivity Scale (SRR)}

\author{
Itai Bermúdez Oviedo ${ }^{1}$, Mirna García Méndez ${ }^{2}$ y Rivera Aragón Sofía ${ }^{3}$
}

\begin{abstract}
Resumen
El rechazo es una experiencia angustiante a la que cada persona reacciona de forma distinta, pues algunas son más sensibles para percibirlo que otras. La sensibilidad genera ansiedad y fácil percepción de un rechazo que podría ser real o imaginario y al que se reacciona de forma extrema. El objetivo del estudio fue diseñar y validar la Escala de Sensibilidad al Rechazo Amoroso (SRA), mediante dos fases; En la fase uno se buscó conocer el significado de la sensibilidad al rechazo amoroso en adultos, en la fase dos se realizó el análisis factorial exploratorio de la escala. Los resultados revelan tres dimensiones del constructo: reacciones afectivas, anticipación y reacciones cognitivas. Se sugiere que estos factores se encuentran determinados por la cultura, se argumentan los hallazgos y se discute su relevancia.
\end{abstract}

Palabras clave: rechazo, sensibilidad al rechazo, rechazo amoroso, pareja, adultos

\begin{abstract}
Rejection is a distressing experience to which each person reacts differently, as some are more sensitive to it than others. Sensitivity generates anxiety and easy perception of a rejection that could be real or imaginary and to which one reacts in an extreme way. The aim of the study was to design and validate the Scale of Sensitivity to Romantic Rejection (SRR), through two phases; First, we focused on understanding the meaning of sensitivity to romantic rejection. Second, we performed an exploratory factor analysis of the scale. The results show three dimensions: affective reactions, anticipation and cognitive reactions. It is suggested that these factors are determined by culture, the findings are argued, and their relevance is discussed.
\end{abstract}

Keywords: rejection, sensitivity to rejection, loving rejection, couple, adults

\footnotetext{
${ }^{1}$ Doctorante en Psicología Social. Universidad Nacional Autónoma de México, Facultad de Estudios Superiores Zaragoza. Av. Guelatao No. 66 (Eje 7 Oriente). Colonia Ejército de Oriente. Ciudad de México. C.P.09230, México. Tel.: 0445527831622. Correo: itai.berovi@gmail.com

${ }^{2}$ Doctora en Psicología Social, Profesora Titular "C" Tiempo Completo. Universidad Nacional Autónoma de México, Facultad de Estudios Superiores Zaragoza. Av. Guelatao No. 66 (Eje 7 Oriente). Colonia Ejército de Oriente. Ciudad de México. C.P.09230.Edificio de Gobierno Planta alta, Carrera de Psicología. Tel.: 56230553. Correo: mina@unam.mx

3 Doctora en Psicología Social, Profesora Titular "C" Tiempo Completo. Universidad Nacional Autónoma de México, Facultad de Psicología. Circuito Ciudad Universitaria Avenida, C.U. 04510 Ciudad de México. Mezzanine, Edificio "D" Tel.: 56222259.Correo: sofiar@unam.mx

Revista Iberoamericana de Diagnóstico y Evaluación - e Avaliação Psicológica. RIDEP · No60 · Vol.3 · 169-181 · 2021

ISSN: 1135-3848 print/2183-6051online
} 


\section{Introducción}

Los seres humanos buscan establecer relaciones para experimentar cariño, cuidado, comodidad, apoyo, amor y comprensión (Rohner \& Carrasco, 2014). Sin embargo, en toda relación interpersonal existe la posibilidad de que el rechazo ocurra, y este es considerado como una de las experiencias más aversivas que existen (Leary, 2006) debido a que la mayoría de las emociones que genera están asociadas con infelicidad, dolor y desilusión, lo que conlleva a una disminución del bienestar personal (Baumeister \& Leary, 1995; Leary, Koch, \& Hechenbleikner, 2006). El rechazo, aunque es aversivo, forma parte de la vida y se manifiesta en diversos tipos de relación: laboral, familiar, escolar o de pareja, entre otras (Downey, Bonica, \& Rincon, 1999, Leary, 2006; Rohner \& Carrasco, 2014).

En las relaciones interpersonales, la retirada de atención, cariño, cuidado, comodidad, apoyo y amor pueden ser interpretadas como rechazo. Al respecto la teoría de aceptación-rechazo interpersonal (Rohner \& Carrasco, 2014), refiere cuatro dimensiones (1) frialdad o falta de afecto, que se manifiesta de forma física y verbal con la ausencia de besos, abrazos, y arrumacos, así como con la falta de elogios, cumplidos, mensajes positivos y halagos; (2) hostilidad o agresividad, que implica golpes, patadas, arañazos, pellizcos, empujones, sarcasmo, maldiciones, así como comentarios desagradables y crueles; (3) indiferencia o negligencia, que tiene que ver con la falta de disponibilidad tanto física como psicológica y con la poca atención a las necesidades de los demás; y (4) rechazo indiferenciado, que implica la creencia de no ser aceptado por los otros, incluso cuando no existen indicadores conductuales que lo demuestren.

Las personas que han sido rechazadas experimentan dolor (Eisenberg, Matthew, Lieberman, \& Williams, 2003), enojo, agresión, hostilidad (Baumeister, Brewer, Tice, \& Twenge, 2007; Bisimi, 2017; Leary, et al., 2006, Rohner \& Carrasco, 2014) tristeza, soledad, celos, culpa, pena, vergüenza y ansiedad. Así mismo, muestran una tendencia a promover la aceptación - por ejemplo, haciendo favores -, conformándose con lo que el otro desee dar con tal de no ser rechazados, o bien hacen todo lo posible por aparentar ser personas socialmente deseables (Fisher, 2006; Leary et al., 2006). Los individuos sensibles al rechazo también suelen ser muy complacientes debido a que buscan mantener la estabilidad en sus relaciones, aunque implique hacer cosas que no quieren, ocultar lo que desean con tal de sentirse aceptados, o bien, pueden sentir cierta devaluación personal ante la idea de ser rechazados por otros (Nezlek, Kowalski, Leary, Blevins, \& Holgate, 1997). De manera contraria, también se ha demostrado que hay personas que cuando perciben el rechazo tienden a ser más agresivas y destructivas al considerar que los otros los rechazan sin razón aparente, por lo que buscan vengarse experimentando emociones con valencia afectiva desagradable (Kelly, 2006; Leary, et al. 2006; Rosales-Saravia, Rivera, Martinez, \& García, 2017).

Por otra parte, se reportan diferencias importantes al percibir y reaccionar ante el rechazo, mientras que para unas personas puede ser intrascendente y se mantienen serenas y despreocupadas, otras se muestran ansiosas y preocupadas (Downey \& Feldman, 1996; Rohner \& Carrasco, 2014). De tal modo, es importante resaltar que no es el rechazo en sí, lo que determina los efectos negativos en el bienestar, sino las percepciones propias que cada individuo tiene sobre su ocurrencia (Colman, 1971).

Para explicar las diferencias de percibir y reaccionar ante el rechazo, surge el término sensibilidad al rechazo, definido como la tendencia a esperar ansiosamente, percibir fácilmente y reaccionar de forma intensa ante el rechazo (Downey \& Feldman, 1996). Este término permite identificar la posibilidad de que una persona se sienta rechazada incluso en situaciones ambiguas en las que el rechazo podría ser imaginario, debido a la hipervigilancia de las acciones de los otros. Se sabe que una persona sensible al rechazo tiende a anticipar sus reacciones y acciones pues para ellos no es necesario que este ocurra, basta con imaginarse que puede ocurrir, pues interpretan el comportamiento insensible de los otros como evidencia de que la rechazan intencionalmente, aun ante la ambigüedad de la situación (Colman, 1971; Downey \& Feldman, 1996; Levy, Ayduk, Downey, \& Leary, 2006). Las personas que son muy sensibles al rechazo interpretan incluso los 
menosprecios menores y el comportamiento insensible de los otros como evidencia de que los rechazan (Downey \& Feldman, 1996; Levy et al., 2006).

Como parte de las emociones con valencia afectiva desagradable que experimentan las personas sensibles al rechazo están el enojo y la tristeza, seguidas de la manifestación de celos, conductas controladoras, hostilidad, soledad, aislamiento y retirada de apoyo (Downey \& Feldaman, 1996; Romero-Canyas, Downey, Berson, Ayduk, \& Kang, 2010; Leary, Koch, \& Hchenbleikner, 2006; Levy et al., 2006; London, Downey, \& Bonica, 2007). Estas personas tienen mayores probabilidades de presentar problemas en su salud física y mental, debido a que son más propensos a padecer depresión, trastornos alimenticios y de la personalidad, ansiedad social, baja autoestima, así como susceptibilidad al estrés y suicidio (Cardi, Di Matteo, \& Corfield, 2013; Kelly, 2001; Nezlek et al., 1997; Rosenbach, 2013; Watson \& Nesdale; 2012).

Las respuestas ante el rechazo varían en forma y función, debido a que puede haber personas que presenten conductas antisociales (agresivas, hostiles, entre otras) o bien pro sociales (acciones que motivan a la mejora de la relación), las segundas como intentos directos o indirectos de recuperar la armonía en las relaciones (Sunami, Nazdan, \& Jaremka, 2019).

El modelo de Levy et al. (2006) explica la forma en que surgen los pensamientos, sentimientos y comportamientos en situaciones sociales en las que es percibido el rechazo. Propone que la sensibilidad al rechazo está compuesta por las experiencias de rechazo que se asocian con la historia previa de interacción, las cuales provocan que ante cualquier estímulo amenazante se manifieste la percepción de rechazo, la cual a su vez provoca reacciones cognitivas $y$ afectivas que generan diversas reacciones conductuales por parte de quien se siente rechazado. Los cinco componentes citados (experiencias, estímulo, percepción, reacciones cognitivas y afectivas, y reacciones conductuales) llevan a que la pareja se aleje de quien es sensible al rechazo, de tal manera que las expectativas se hacen reales, lo que se convierte en una profecía autocumplida que termina por reforzar las experiencias y hace que se repita el ciclo en otras relaciones.

El rechazo amoroso es uno de los eventos de vida más estresantes en los adultos asociado a distintos patrones de síntomas afectivos (Hsu, Sankar, Malik, Langenecker, Mickey, \& Love, 2020). De tal manera que la sensibilidad al rechazo también se asume como un proceso cognitivo-afectivo (Downey \& Feldman, 1996; Nomannsell \& Wisco, 2017) conformado por una evaluación relacional percibida baja, lo que significa que quien es sensible al rechazo siente que no es del todo valioso, importante o cercano para los otros (Leary, 2006).

El término sensibilidad al rechazo ha sido estudiado de manera general en la familia, en el trabajo, en la escuela, con los amigos y con la pareja (Bringle, Winnick, \& Rydell, 2013; Downey \& Feldman, 1996; Rosenbach, 2013). Sin embargo, resulta importante tomar en cuenta la influencia cultural, el tipo de relación que se tiene, e incluso las características de cada individuo (Baumeister \& Leary, 1995; Bonilla-Algovia \& Rivas-Rivero, 2020; Leary, 2006; Rohner \& Carrasco, 2014).

Diversos hallazgos empíricos identifican variables que matizan la sensibilidad al rechazo amoroso, tal es el caso de los estilos de apego adulto. Las personas con apego ansioso son más sensibles al rechazo que aquellas con apego seguro y son las primeras quienes tienen mayores dificultades en sus relaciones interpersonales (Downey \& Feldman, 1996; Sato, Fonagy, \& Luyten, 2020; Mandal \& Latusek, 2014). Por ora parte, la personalidad puede considerarse un predictor sobre qué tan sensible al rechazo pude ser un individuo (Cavicchioli \& Maffei, 2020; Brookings, Zembar, \& Hochstetler, 2003). De igual manera, se ha demostrado que una persona codependiente percibe fácilmente el rechazo, ya que necesita sentirse aceptada, valorada e incluida (Chang, 2012). Asimismo, cuando una persona tiene problemas de autoestima, suele ser sensible al rechazo, ya que la percepción de ser poco valiosa o importante la mantiene excesivamente alerta de las acciones de los demás volviéndose vulnerables (Zhou, Li, Tian, \& Huebner, 2020). Todo esto permite considerar que la sensibilidad al rechazo puede ser causa o consecuencia de diversos problemas que afectan la salud mental, por lo que es relevante su estudio y medición. 
Actualmente existen diversas formas de medir la sensibilidad al rechazo, la herramienta más utilizada en las investigaciones internacionales es el Cuestionario de Sensibilidad al Rechazo (RSQ) por sus siglas en inglés, diseñado por Downey y Feldman (1996), consta de 18 reactivos que miden el grado de preocupación o ansiedad ante el rechazo, así como las expectativas de aceptación o rechazo en diversos tipos de relaciones. Se ha traducido y adaptado para diferentes contextos y diversas poblaciones, por ejemplo, se elaboró el Cuestionario de Sensibilidad al Rechazo para Niños (CRSQ) (Downey, Lebolt, Rincón, \& Freitas, 1998), se adaptó el cuestionario para población hindú (Bishmi, 2017) y se realizó una adaptación particularmente para niños Coreanos (Sang-Hwang, 2012).

La escala de Sensibilidad al Rechazo Basada en la Apariencia (Park, 2007) es una adaptación de los reactivos de la RSQ particularmente enfocados al rechazo basado en el atractivo físico percibido. De forma similar se adaptó el RSQ para población homosexual, considerando la sensibilidad al rechazo particularmente en las relaciones familiares (Pachankis, 2008).

Por otra parte, Bianchi, Schonfeld y Laurent (2015) diseñaron un reactivo único que les permitiera evaluar el temor recurrente de ser rechazado, juzgado o criticado por los otros. Lo que consiguieron con este reactivo fue identificar relaciones interpersonales tormentosas, incapacidad para iniciar o mantener relaciones, y el miedo generalizado que las personas tienen a sentir vergüenza. También se elaboró la escala de Sensibilidad al Rechazo para población adolescente para identificar las estrategias de afrontamiento que tienen los jóvenes ante el rechazo (Zimmer-Gembeck \& Skinner, 2015).

En este punto es importante resaltar que la sensibilidad al rechazo varia de una relación a otra, y que cuanto más significativas sean estas relaciones, más dolorosa será la experiencia pues dependiendo de la intensidad del vínculo, serán más o menos intensas las reacciones que se tengan ante él (Leary, 2006), así como la percepción que se tenga de él (Downey \& Feldman, 1996). Es así que, al ser las relaciones de pareja de las más significativas que una persona establece (Regan, 2011), resulta importante analizar lo que ocurre con la sensibilidad al rechazo particularmente en la relación de pareja lo que no ha sido evaluado en los cuestionarios señalados anteriormente.

La sensibilidad al rechazo puede intensificarse en las relaciones de pareja, ya que en estas aumenta la vulnerabilidad al rechazo y al dudar que alguna vez serán aceptados por la persona amada, pueden responder de formas contraproducentes, evitando oportunidades de interacciones potencialmente valiosas (Downey \& Daniels, 2020).

Los resultados negativos asociados con la sensibilidad al rechazo son múltiples para las relaciones interpersonales, de manera particular estas personas se muestran más ansiosas en la relación, con una mayor preocupación por su futuro, centrándose en los eventos y en las interacciones negativas cargadas de angustia e incluso desgaste físico resultado de la evaluación relacional percibida de manera negativa (Cardi, Di Matteo, \& Corfield, 2013; Nowland, Talbot, \& Qualter, 2018). Por lo que es importante establecer un instrumento de medición para la sensibilidad al rechazo amoroso.

Las personas que son sensibles al rechazo amoroso interpretan incluso los menosprecios menores y el comportamiento insensible de su pareja como evidencia de que los rechazan, enfocan su atención de forma sesgada hacia situaciones que confirme que están siendo rechazados, lo que genera trastornos cognitivos, conductuales y emocionales, como depresión, conductas de riesgo, procrastinación - pues quien teme ser rechazado posterga sus actividades para evitar correr riesgos de rechazo - (Watson \& Nesdale, 2012), enojo, agresión y hostilidad -que a su vez pueden convertirse en violencia (Baumeister, Brewer, Tice, \& Twenge, 2007; Leary et al., 2006; Rohner \& Carrasco, 2014), tristeza, soledad, dolor, celos, culpa, pena vergüenza y ansiedad (Fisher, 2006; Leary et al., 2006; Nezlek et al., 1997;). Todo esto se asocia con reacciones más graves y efectos negativos más duraderos ante el rechazo como pueden ser asesinatos y suicidios (Kraines, White, Grant, \& Wells, 2019; Cain, De Panfilis, Meehan, \& Clarkin, 2017).

Con base en lo descrito y en las características de las escalas mencionadas, el objetivo de esta investigación fue realizar un instrumento válido, confiable y culturalmente relevante que mida la 
sensibilidad al rechazo amoroso en adultos, considerando elementos teóricos y evidencia empírica (Downey \& Feldman 1996; Levy et al., 2006). Para dar cumplimiento al objetivo planteado, la investigación se llevó a cabo en dos fases.

\section{Fase1}

La sensibilidad al rechazo varía en cada persona, por lo que hay quienes pueden permanecer serenos y sin problemas cuando sus parejas los rechazan y quienes al ser más sensibles se ven más afectados (Ayduck \& Gyurak, 2008; Downey \& Feldman, 1996). Por otra parte, es importante considerar que la mayoría de los estudios que se han hecho en torno a esta variable están enfocados a la ansiedad y preocupación de que el rechazo ocurra, así como a las expectativas de aceptación o rechazo por parte de los otros, sin embargo, es importante conocer cómo conceptúan los adultos la sensibilidad al rechazo amoroso, lo cual es el objetivo de esta fase, en la que se realizará un análisis de contenido.

\section{Método}

\section{Participantes}

Colaboraron 100 adultos (52\% mujeres y $48 \%$ hombres) de la ciudad de México y zona metropolitana con un rango de edad de 20 a 40 años $(M=24.81, D E=5.44)$ con el criterio de inclusión de que estuvieran en una relación de pareja de por lo menos un año de duración. La muestra fue no probabilística intencional.

\section{Instrumento}

Cuestionario de preguntas abiertas sobre sensibilidad al rechazo amoroso (Anexo 1). Se elaboró un cuestionario de 6 preguntas abiertas, sustentadas en el modelo de sensibilidad al rechazo (Levy et al., 2006), el cual incluyó enunciados relacionados con percepciones, reacciones cognitivas, reacciones afectivas y reacciones conductuales ante el rechazo.

\section{Procedimiento}

Se aplicó el cuestionario en espacios públicos de la Ciudad de México y zona Metropolitana, a los participantes que cumplieron con los criterios de inclusión y que aceptaron participar en el estudio de manera voluntaria, se les informó sobre el anonimato y confidencialidad de la información recabada y se recolectaron las firmas de consentimiento. Al finalizar la aplicación se agradeció a los participantes su colaboración. Los datos obtenidos se transcribieron con ayuda del programa Excel, en el que se organizaron y analizaron las respuestas.

\section{Resultados}

Se realizó un análisis de contenido, el cual permite considerar categorías basadas en la teoría, y establecidas a priori del análisis (Mayring, 2014). Cada categoría se obtuvo a partir de las palabras con mayor frecuencia en las respuestas de los participantes (ver Tabla 1). Los resultados se agruparon en cuatro categorías coincidentes con el modelo de Levy et al. (2006): percepción, reacciones cognitivas, reacciones afectivas y reacciones conductuales, adicionalmente se identificó la categoría de anticipación que implica prever el rechazo de la pareja, evitando acciones como el romanticismo, o las peticiones, por temor al rechazo. Esta categoría es consistente con diversas investigaciones empíricas (Colman, 1971; Downey \& Feldman, 1996; Levy, Ayduk, Downey, \& Leary, 2006).

A partir de los resultados del análisis de contenido y de la teoría sobre sensibilidad al rechazo, se redactaron 83 reactivos que integraran la información recolectada en esta fase y que fueron revisados por cinco expertos en investigación sobre relaciones de pareja, quienes hicieron observaciones de contenido al cuestionario.

\section{Fase 2}

El Cuestionario de Sensibilidad al Rechazo (Downey \& Felman, 1996) es el instrumento más utilizado en las investigaciones sobre este fenómeno, y ha sido validado en diversos países y para diferentes poblaciones, sin embargo, se enfoca a la sensibilidad al rechazo en cualquier tipo de relación y mide las expectativas de aceptación y rechazo, así como la ansiedad que estas generan. Es necesario realizar un instrumento válido, confiable $\mathrm{y}$ culturalmente 
Tabla 1. Categorías e indicadores de sensibilidad al rechazo amoroso $(\mathrm{N}=100)$

\begin{tabular}{|c|c|c|c|}
\hline Categoría & Definición & Indicadores & $\mathrm{f}$ \\
\hline Percepción & $\begin{array}{l}\text { Identificar rechazo amoroso intencional } \\
\text { en las conductas ambiguas de la pareja. }\end{array}$ & $\begin{array}{l}\text { Mi pareja no me muestra interés, no quiere estar conmigo, es } \\
\text { indiferente, me ignora, no es afectuoso, parece fastidiado de } \\
\text { estar conmigo, no me pone atención, no es cuidadoso conmigo, } \\
\text { me evita, no quiere tener relaciones sexuales, se aleja. }\end{array}$ & 167 \\
\hline $\begin{array}{l}\text { Reacciones } \\
\text { cognitivas }\end{array}$ & $\begin{array}{l}\text { Explicaciones personales que surgen ante } \\
\text { los motivos del rechazo amoroso y que } \\
\text { determinan las respuestas que se tienen } \\
\text { ante el mismo. }\end{array}$ & $\begin{array}{l}\text { Mi pareja me rechaza porque no quiere estar conmigo, no le } \\
\text { intereso, no soy importante para ella, hago algo que le molesta, } \\
\text { lo desespero, no se le da expresar sus sentimientos, hice algo } \\
\text { que no le gustó, se siente sofocada por mí, se harta de mí, se } \\
\text { siente abrumado por mí. }\end{array}$ & 237 \\
\hline $\begin{array}{l}\text { Respuestas } \\
\text { afectivas }\end{array}$ & $\begin{array}{l}\text { Conjunto de sentimientos y emociones } \\
\text { que surgen ante la percepción de rechazo } \\
\text { amoroso. }\end{array}$ & $\begin{array}{l}\text { Tristeza, dolor, miedo, ansiedad, culpa, malestar, desilusión, } \\
\text { inseguridad, angustia, humillación, desesperación, melancolía, } \\
\text { ganas de llorar, enojo. }\end{array}$ & 253 \\
\hline $\begin{array}{l}\text { Respuestas } \\
\text { conductuales }\end{array}$ & $\begin{array}{l}\text { Conjunto de acciones que surgen como } \\
\text { respuesta ante la percepción del rechazo } \\
\text { amoroso. }\end{array}$ & $\begin{array}{l}\text { Me alejo, lloro, soy más cariñoso(a), me pongo triste, le doy su } \\
\text { espacio, evito pelear, me quedo callada, no hago nada, me siento } \\
\text { mal, me siento frustrada, yo también lo rechazo, me da ansiedad. }\end{array}$ & 185 \\
\hline
\end{tabular}

relevante que mida de forma particular la sensibilidad al rechazo amoroso en adultos, permitiendo la obtención de datos empíricos que fortalezcan el conocimiento sobre la sensibilidad al rechazo amoroso.

\section{Método}

\section{Participantes}

La muestra fue seleccionada mediante un muestreo no probabilístico intencional y estuvo constituida por 415 adultos voluntarios de la ciudad de México, 136 (32.8\%) hombres y 279 (67.2\%) mujeres, con edades comprendidas entre los 20 y 40 años $(M=26, D E=6.92)$, con una relación de pareja de por lo menos un año y que en algún momento se sintieron rechazados en dicha relación, en la Tabla 2 se presentan los datos sociodemográficos.

\section{Instrumentos}

\section{Escala de Sensibilidad al Rechazo Amoroso} (SRA). Con los resultados de la fase 1 y tomando en cuenta la teoría (Levy et al., 2006), se elaboró una escala conformada por 83 reactivos con formato de respuesta tipo Likert con cinco opciones de respuesta que van de $1=$ Totalmente en desacuerdo, 2=En desacuerdo, $3=\mathrm{Ni}$ de acuerdo ni en desacuerdo, 4=De acuerdo, $5=$ Totalmente de acuerdo.

Escala de Estilos de Apego Adulto. (Márquez, Rivera, \& Reyes, 2009), integrada por 21 reactivos $(\alpha=.74)$ con 7 opciones de respuesta: (1=totalmente en desacuerdo y $7=$ totalmente de acuerdo), agrupados en 3 factores: apego evitante $(\alpha=.88)$, apego ansioso $(\alpha=.86)$ y apego seguro $(\alpha=.81)$, que explican el $55.49 \%$ de la varianza. Esta se aplicó a la misma muestra, con el propósito de obtener correlaciones con la escala de Sensibilidad al Rechazo Amoroso que se obtenga como resultado de esta fase.

\section{Procedimiento}

Se aplicaron las escalas en espacios públicos de la Ciudad de México y zona Metropolitana, así como en formato digitalizado a personas que tenían una relación de pareja de por lo menos un año, que en dicha relación se hubiesen sentido rechazados y que aceptaron participar en el estudio. Se les informó que su participación era voluntaria, anónima, confidencial y que sus datos serían utilizados con fines de investigación, para posteriormente recolectar el consentimiento informado por participante. Los datos recolectados fueron analizados en el programa SPSS versión 20.

\section{Análisis de datos}

Para la discriminación de reactivos se realizó una distribución de frecuencias para identificar el porcentaje obtenido en cada una de las opciones de respuesta. Se calculó el sesgo para conocer la distribución de los reactivos. Se obtuvo la correlación reactivo total donde todos los reactivos obtuvieron valores mayores a .50 y se aplicó una prueba $t$ de Student para grupos extremos, se 
Tabla 2. Características sociodemográficas de la muestra $(\mathrm{n}=415)$

\begin{tabular}{lcc}
\hline & $\mathrm{f}$ & $\%$ \\
\hline Sexo & 279 & $67.2 \%$ \\
Mujer & 136 & $32.8 \%$ \\
Hombre & & \\
Estado civil & 226 & $54.5 \%$ \\
Noviazgo & 94 & $22.7 \%$ \\
Casados & 86 & $20.7 \%$ \\
Unión libre & 9 & $2.2 \%$ \\
Otro & & \\
Escolaridad & 186 & $44.8 \%$ \\
Preparatoria & 111 & $26.7 \%$ \\
Licenciatura & 64 & $15.4 \%$ \\
Técnico & 43 & $10.4 \%$ \\
Secundaria & 6 & $1.4 \%$ \\
Posgrado & 5 & $1.2 \%$ \\
Primaria & & \\
Ocupación & 171 & $41.2 \%$ \\
Estudiante & 102 & $24.6 \%$ \\
Ama de casa & 91 & $21.9 \%$ \\
Oficio & 51 & $12.3 \%$ \\
Profesionista & &
\end{tabular}

Tabla 3. Ponderación de factores con rotación oblimin de la Escala de Sensibilidad al Rechazo Amoroso (SRA) $(\mathrm{N}=415)$

\begin{tabular}{|c|c|c|c|}
\hline \multirow{2}{*}{ Reactivos } & \multicolumn{3}{|c|}{ Factor } \\
\hline & 1 & 2 & 3 \\
\hline 28.Me siento ansioso(a) cuando noto que mi pareja me está evitando & .920 & .055 & -.121 \\
\hline 46.Cuando creo que mi pareja me está evitando me siento inseguro(a) & .907 & -.036 & -.026 \\
\hline 74.Cuando creo que mi pareja se niega a estar conmigo me siento desesperado(a) & .771 & .100 & -.026 \\
\hline 78. Cuando creo que mi pareja se niega a estar conmigo me siento triste & .698 & -.031 & .148 \\
\hline 22.Cuando percibo que mi pareja me está evitando siento miedo & .703 & .001 & .112 \\
\hline 40.Me duele sentir que mi pareja se niega a estar conmigo & 669 & -.047 & .237 \\
\hline 11.Cuando percibo que mi pareja me evita siento culpa & .660 & .074 & .044 \\
\hline 34.Me siento mal cuando considero que mi pareja se niega a estar conmigo & .606 & .058 & .187 \\
\hline 17.Me siento humillado(a) cuando parece que mi pareja me evita & .553 & .279 & .054 \\
\hline 19.Evito ser detallista con mi pareja porque siento que va a ignorar mis detalles & -.149 & .831 & .095 \\
\hline 7.Evito etiquetar a mi pareja en redes sociales porque siento que va a ignorarme & .061 & .766 & -.135 \\
\hline 60.Evito decirle a mi pareja que la quiero por temor a NO ser correspondido(a) & -.004 & .732 & .064 \\
\hline 54.Evito pedirle a mi pareja que salgamos juntos por temor a que se niegue & .107 & .653 & .079 \\
\hline 2.Evito hacer cosas por mi pareja porque siento que NO va a corresponderme & -.025 & .628 & .055 \\
\hline 13.Evito pedirle cosas a mi pareja porque siento que va a negarse & .094 & .606 & .101 \\
\hline 48.Le oculto mis problemas a mi pareja porque siento que se negará a apoyarme & .133 & .578 & .059 \\
\hline 30.Evito pedirle a mi pareja que tengamos relaciones porque siento que va a negarse & .163 & .553 & .027 \\
\hline 44.Pienso que mi pareja me evita porque dejé de cumplir sus expectativas & -.049 & .029 & .870 \\
\hline 21.Pienso que mi pareja se niega a estar conmigo porque hago cosas que le molestan & .137 & -.097 & .730 \\
\hline 77.Creo que mi pareja se niega a estar conmigo porque parece fastidiada & -.003 & .075 & .754 \\
\hline 56. Creo que mi pareja se niega a estar conmigo porque la abrumo con mi cariño & -.032 & .116 & .709 \\
\hline 32.Pienso que mi pareja se aparta de mí porque lo desespero & .056 & .034 & 694 \\
\hline 15.Pienso que mi pareja me aparta porque le cuesta expresar sus sentimientos & .059 & .039 & .568 \\
\hline 71.Creo que si mi pareja evita escucharme es porque le importo poco & .164 & .122 & .529 \\
\hline Número de reactivos & 9 & 8 & 7 \\
\hline Porcentaje de varianza explicada & $51.46 \%$ & $5.21 \%$ & $3.58 \%$ \\
\hline Alfa de Cronbach & .94 & .90 & .90 \\
\hline
\end{tabular}

Nota. Los números en negrita representan las cargas factoriales más altas

consideró como criterio de eliminación los reactivos que tuvieran una significancia <.05. Se obtuvieron los valores de la medida de adecuación muestral de Kaiser-Meyer-Olkin (KMO) y de la prueba de esfericidad de Bartlett, a partir de valores de entre .05 y 1 con una fiabilidad menor a .05 respectivamente. Estos parámetros indican que tan apropiado es aplicar el análisis factorial y se comprueba que la matriz de correlaciones es de identidad (Suárez, 2007). Posteriormente se llevó a cabo el análisis psicométrico mediante un análisis factorial exploratorio de ejes principales com rotación oblimin para obtener la estructura factorial de los reactivos y las dimensiones de la escala. Sólo fueron considerados los reactivos 
Tabla 4. Definición de los factores de la Escala de Sensibilidad al Rechazo Amoroso (SRA)

\begin{tabular}{lll}
\hline Factor & Definición & Indicadores \\
\hline $\begin{array}{l}\text { 1.Reacciones } \\
\text { afectivas }\end{array}$ & $\begin{array}{l}\text { Expresión de sentimientos y emociones relacionados con la } \\
\text { sensación de rechazo por parte de la pareja. }\end{array}$ & $\begin{array}{l}\text { Sentir malestar, culpa, inseguridad, } \\
\text { tristeza, dolor, miedo y humillación ante } \\
\text { la idea de ser rechazado por la pareja. }\end{array}$ \\
$\begin{array}{lll}\text { 2. Anticipación } \\
\text { Prever la situación y evitar hacer cosas debido a que se espera } \\
\text { que la se niegue a dar o recibir cualquier muestra de amor, carino de hacer, pedir o decir cosas por } \\
\text { yeacciones } \\
\text { cognitivas }\end{array}$ & Pensamientos y creencias que surgen ante el rechazo de la pareja. & $\begin{array}{l}\text { Considerar que la pareja se siente harta, } \\
\text { molesta, abrumada o desesperada. }\end{array}$ \\
\hline
\end{tabular}

con peso factorial igual o mayor a 40 lo que se considera un valor razonable debido a que indica la representatividad del reactivo en cada factor (Suárez, 2007). Por último, se realizó la prueba Alpha de Conbrach, para obtener los índices de consistencia interna por factor, así como la confiabilidad global del instrumento.

\section{Resultados}

De acuerdo con el análisis de los reactivos según su frecuencia, sesgo, correlación y discriminación, ninguno obtuvo criterios de eliminación para ser descartados. Se obtuvieron los valores de la medida de adecuación muestral de Kaiser-Meyer-Olkin $(K M O)=0.96$ y de la prueba de esfericidad de Bartlett $X^{2}(7156.31)=276, p<.001$, con lo que se asume que los datos provienen de una distribución normal multivariante. Se aplicó el análisis factorial exploratorio con ejes principales y rotación oblimin a los 83 reactivos de la escala. Se eliminaron en total 59 reactivos debido a que 13 se distribuyeron en factores con menos de tres reactivos y 46 porque tenían pesos factores mayores a .40 en más de un factor (Lloret, Ferreres, Hernández, \& Tomás, 2014), lo que dio como resultado un instrumento compuesto de 24 reactivos distribuidos en tres factores que explican el $60.26 \%$ de la varianza total con un Alfa de Cronbach global de .96 (ver Tabla 3). Las definiciones e indicadores de los factores se presentan en la Tabla 4.

Posteriormente se obtuvo la correlación (ver Tabla 5) entre los tres factores. Como se puede observar, todas son altas y positivas.

De acuerdo con los hallazgos de este estudio se propone definir la sensibilidad al rechazo amoroso como la tendencia a percibir y esperar el rechazo de la pareja como resultado de hipervigilar sus acciones, reaccionando con afecto
Tabla 5. Correlaciones de los factores de sensibilidad al rechazo

\begin{tabular}{lccc}
\hline & $\begin{array}{c}\text { Reacciones } \\
\text { afectivas }\end{array}$ & Anticipación & $\begin{array}{c}\text { Reacciones } \\
\text { cognitivas }\end{array}$ \\
\hline $\begin{array}{l}\text { Reacciones } \\
\text { afectivas }\end{array}$ & 1 & $.709^{* * *}$ & $.785^{* *}$ \\
$\begin{array}{l}\text { Anticipación } \\
\text { Reacciones } \\
\text { cognitivas }\end{array}$ & 1 & $.728^{* *}$ \\
$* *$ Correlación estadísticamente significativa al .01.
\end{tabular}

Tabla 6. Correlaciones entre la escala de Sensibilidad al rechazo y la de Estilos de Apego

\begin{tabular}{lccc}
\multicolumn{4}{c}{ Adulto } \\
\hline $\begin{array}{c}\text { Apego } \\
\text { evitante }\end{array}$ & $\begin{array}{c}\text { Apego } \\
\text { ansioso }\end{array}$ & $\begin{array}{c}\text { Apego } \\
\text { seguro }\end{array}$ \\
\hline $\begin{array}{l}\text { Reacciones } \\
\text { cognitivas }\end{array}$ & $.310^{* *}$ & $.619^{* *}$ & $-.401^{* *}$ \\
$\begin{array}{l}\text { Anticipación } \\
\text { Reacciones } \\
\text { afectivas }\end{array}$ & $.333^{* *}$ & $.567^{* *}$ & $-.426^{* *}$ \\
\hline
\end{tabular}

** Correlación estadísticamente significativa al .01.

negativo y anticipándose a que suceda, lo que ocasiona reacciones cognitivas devaluativas.

Se continuó con un análisis de validez convergente con los factores de la Escala de Estilos de Apego Adulto de Márquez-Domínguez et al. (2009). Las correlaciones obtenidas más fuertes y positivas, son las de reacciones cognitivas y reacciones afectivas con el apego ansioso, seguidas por las correlaciones de anticipación con el apego ansioso, lo que sugiere que la sensación de tristeza y culpa, así como sentirse abrumado o desesperado son factores que facilitan manifestaciones de apego ansioso en las personas (ver Tabla 6). Por el contrario, las correlaciones negativas con el apego seguro sugieren que puede ser un factor protector para que disminuya en las personas la sensibilidad al rechazo amoroso. 


\section{Discusión}

El objetivo del estudio fue elaborar una escala para medir la sensibilidad al rechazo amoroso, se obtuvieron tres factores: reacciones afectivas, anticipación y reacciones cognitivas, los cuales fueron definidos en función de sus indicadores, aportando conocimiento empírico sobre la variable de interés.

En el análisis factorial, a diferencia del modelo de Levy et al. (2006), los factores de percepción y reacciones conductuales no tuvieron el peso factorial suficiente para mantener los reactivos en la escala, lo cual coincide con lo señalado por Downey y Feldmand (1996), quienes indicaron que la sensibilidad al rechazo consiste en percibir fácilmente el rechazo en las acciones de los otros, la percepción es generalizada, y surge de las experiencias previas de las personas (Levy et al., 2006) por lo que puede identificarse en los reactivos de la escala.

Las reacciones afectivas (factor 1) tienen el mayor peso factorial, a diferencia de los resultados encontrados en otros estudios (Baumeister et al., 2007; Bishimi, 2017; Leary et al., 2006, Rohner \& Carrasco, 2014), predominan los sentimientos de tristeza, dolor, miedo, inseguridad, ansiedad y culpa, más que sentir enojo, hostilidad o agresividad.

Los indicadores que conforman la anticipación (factor 2) se caracterizan por esperar que la pareja rechace las muestras de afecto, esto apoya los elementos considerados en la definición de sensibilidad al rechazo (Colman, 1971; Downey \& Feldman, 1996; Levy et al., 2006) debido a que la persona sensible al rechazo tiende a anticipar sus reacciones y acciones, ya que el simple hecho de imaginar que pueden rechazarlos les genera angustia.

Las reacciones cognitivas (factor 3) permiten identificar la devaluación personal que las personas hacen al percibir el rechazo, lo que concuerda con lo propuesto por Nezlek et al. (1997). Por otra parte, también se confirma que ante el rechazo las personas hacen una evaluación relacional percibida baja (Leary, 2006), esto significa que quienes son sensibles al rechazo sienten que no son del todo valiosos, importantes o cercanos para su pareja, lo que puede ocasionar que tengan explicaciones sobre los motivos del rechazo en las que se colocan como los culpables, lo que los lleva a ejecutar acciones preventivas o de solución que pueden hacer que un rechazo imaginario se haga real, lo cual corresponde con lo reportado por Levy et al. (2006).

Los resultados de esta investigación permiten diferenciar la sensibilidad al rechazo el rechazo per se. Por un lado, el rechazo se interpreta en términos de acciones directas como la retirada de afecto, cariño y atención (Rohner \& Carrasco, 2014). Por el contrario, la sensibilidad al rechazo se asocia con eventos percibidos por la persona que se siente rechazada lo cual coincide con los hallazgos de Colman (1971) y los aportes Downey $\&$ Feldman (1996).

En conclusión, la Escala de Sensibilidad al Rechazo Amoroso es un instrumento con propiedades psicométricas apropiadas que permite evaluar el constructo en las relaciones de pareja, a diferencia de otros instrumentos enfocados a evaluar las expectativas de aceptación o de rechazo como es el caso de la QRS (Downey \& Feldman, 1996). La SRA está orientada en la percepción de rechazo en situaciones comunes que resultan ambiguas para la pareja con manifestaciones de malestar en quienes son altamente sensibles al rechazo amoroso.

\section{Limitaciones y sugerencias}

Para poder generalizar los datos es pertinente ampliar la muestra con diferentes rangos de edad, así como realizar una segunda aplicación mixta, digital y presencial para corroborar las propiedades psicométricas de la escala y poder realizar un análisis confirmatorio de la misma, ya que debido a la pandemia de COVID-19 su aplicación fue de manera digital y no se tuvo la oportunidad de verificar que tanto afecta esto las respuestas de los participantes.

\section{Agradecimientos}

Se agradece al Consejo Nacional de Ciencia y Tecnología (CONACYT) por la beca otorgada al primer autor con número de becario 631358, número de apoyo 482766 y CVU 858162, con la que se financió la presente investigación. 


\section{Referencias}

Ayduk, O., \& Gyurak, A. (2008). Applying the cognitive-affective processing systems approach to conceptualizing rejection sensitivity. Social and Personality Psychology Compass, 2(5), 2016-203.

doi:10.1111/j.1751-9004.2008.00143.x

Baumeister, R., Brewer, L., Tice, D., \& Twenge, J. (2007). Thwarting the need to belong: Understanding the interpersonal and inner effects of social exclusion. Social and Personality Psychology Compass, 1(1), 506520.

https://doi.org/10.1111/j.1751-

9004.2007.00020.x

Baumeister, R., \& Leary, M. (1995). The need to belong: Desire for interpersonal attachments as a fundamental human motivation. Psychological Bulletin, 117(3), 497-529. doi:10.1037/0033-2909.117.3.497

Bianchi, R., Schonfeld, I. S., \& Laurent, E. (2015). Interpersonal rejection sensitivity predicts burnout: A prospective study. Personality and Individual Differences, 75, 216-219.

doi:doi.org/10.1016/j.paid.2014.11.043

Bishmi, P. D. (2017). A comparitive study on level and direction of aggression in high and low rejection sensitive adolescents. Indian Journal of Health \& Wellbeing, 8(7), 729733.

Bonilla-Algovia, E., \& Rivas-Rivero, E. (2020). Diseño y validación de la Escala de Mitos del Amor Romántico. Revista Iberoamericana de Diagnóstico y Evaluación - e Avaliação Psicológica 4(57), 119-136. doi:10.21865/RIDEP57.4.09

Bringle, R. G., Winnick, T., \& Rydell, R. J. (2013). The prevalence and nature of unrequited love. Sage Open, 3(2). doi:10.1177/2158244013492160

Brookings, J., B., Zembar, M., J., \& Hochstetler, G., M. (2003). An interpersonal circumplex/five-factor analysis of the Rejection Sensitivity Questionnaire. Personality and Individual Diferences, 34(3), 449-461. doi:10.1016/S0191-8869(02)00065-X

Cardi, V., Di Matteo, R., Corfield, F., \& Treasure, J. (2013). Social reward and rejection sensitivity in eating disorders: An investigation of attentional bias and early experiences. World J Biol Psychiatr, 14(8), 622-33.

doi:10.3109/15622975.2012.665479

Cain, N. M., De Panfilis, Ch., Meehan, K. V., \& Clarkin, J. F. (2017). A multisurface interpersonal circumplex assessment of rejection sensitivity. Journal of Personality Assessment, 99(1), 35-45. doi:http://dx.doi.org/10.1080/00223891.2016. 1186032

Cavicchioli, M., \& Maffei, C. (2020). Rejection sensitivity in borderline personality disorder and the cognitive-affective personality system: A meta-analytic review. Personality Disorders: Theory, Research and Treatment, 11(1), 1.

Chang, S. H. (2012). A cultural perspective on codependency and its treatment. Asia Pacific Journal of Counselling and Psychotherapy, 3(1), 50-60.

Colman, A. (1971). Social rejection, role conflict, and adjustment: Psychological consequences of orthopedic disability. Perceptual and Motor Skills, 33(3), 907-910. doi:10.2466/pms.1971.33.3.907

Downey, G., Bonica, C., \& Rincon, C. (1999). Rejection sensitivity and adolescent romantic relationships. In Furman W., Brown, B., \& Feiring, C. (Eds.) The development of Romantic Relationships in Adolescence. New York: Cambridge University Press.

Downey, G., \& Daniels, J. E. (2020). The dynamic ecology of rejection and acceptance: Mental health implications. Arch Sex Behav, 49, 2269-2273.

doi:https://doi.org/10.1007/s10508-020-

01636-8

Downey, G., \& Feldman, S. (1996). Implications of rejection sensitivity for intimate relationships. Journal of Personality and Social Psychology, 70(6), 1327-1343. doi:10.1037//0022-3514.70.6.1327

Downey, G., Lebolt, A., Rincón, C., \& Freitas, A. (1998). Rejection Sensitivity and Children's Interpersonal Difficulties. Child Development, 69(4), 1074-1091. doi:10.2307/1132363

Eisenberger, N. I., Lieberman, M. D., \& Williams, K. D. (2003). Does rejection hurt? An fMRI 
study of social exclusion. Science, 302(5643), 290-292. doi:10.1126/science.1089134

Fisher, H. (2006). Lost love. The nature of romantic rejection. In N. Bauer-Maglin (Ed.), Cut Loose: (Mostly) Older Women Talk About the End of (Mostly) Long-Term Relationships (pp. 181-195) New Brunswick: Rutgers University Press.

Hsu, D. T., Sankar, A., Malik, M. A., Langenecker, S. A., Mickey, B. J., \& Love, T. M. (2020). Common neural responses to romantic rejection and acceptance in healthy adults. Social Neuroscience, 15(5), 571-583. doi:10.1080/17470919.2020.1801502

Kelly, M. K. (2006). Individual differences in reactions to rejection. In M. R. Leary (Ed.). Interpersonal Rejection (pp. 291-316). Retrieved from: Oxford Scholarship Online. doi:10.1093/acprof:oso/97801951130157.001. 001

Kraines, M. A., White, E. J., Grant, D. M., \& Wells, T. T. (2019). Social anxiety as a precursor for depression: Influence of interpersonal rejection and attention to emotional stimuli. Psychiatry research, 275, 296-303. doi:10.1016/j.psychres.2019.04.001

Leary, M. (2006). Toward a conceptualization of interpersonal rejection. In M. R. Leary (Ed.). Interpersonal Rejection. (pp. 3-20). Retrieved from: Oxford Scholarship Online. doi:10.1093/acprof:oso/97801951130157.001.0 01

Leary, M. R., Koch, E. J., \& Hechenbleikner, N., R. (2006). Emotional Responses to Interpersonal Rejection. In M. R. Leary (Ed.). Interpersonal Rejection. (pp. 145-166) Retrieved from: Oxford Scholarship Online. doi:10.1093/acprof:oso/97801951130157.001. 001

Levy, S. R., Ayduk, O., Downey, G., \& Leary, M. $\mathrm{R}$. (2006). The role of rejection sensitivity in people's relationships with significant others and valued social groups interpersonal rejection. New York: Oxford University Press.

Lloret-Segura, S., Ferreres-Traver, A., HernándezBaeza, A., \& Tomás-Marco, I. (2014). El análisis factorial exploratorio de los ítems: Una guía práctica, revisada y actualizada. Anales de Psicología/Annals of Psychology,
30(3), 1151-1169.

doi:10.6018/analesps.30.3.199361

London, B., Downey, G., Bonica, C., \& Paltin, I. (2007). Social causes and consequences of rejection sensitivity. Journal of Research on Adolescence, 17(3), 481-506. doi:10.1111/j.1532-7795.2007.00531.x

Mandal, E., \& Latusek, A. (2014). Attachment styles and anxiety of rejecters in intimate relationships. Current Issues in Personality Psychology, 2(4), 185-195.

Mayring, P. (2014). Qualitative Content Analysis: Theoretical Foundation, Basic Procedures and Software Solution. Klagenfurt: Open Access Repository.

Nezlek, J. B., Kowalski, R. M., Leary, M. R., Blevins, T., \& Holgate, S. (1997). Personality moderators of reactions to interpersonal rejection: Depression and trait self-esteem. Personality and Social Psychology Bulletin, 23(12), 1235-1244. doi:10.1177/01461672972312001

Nomannsell, K. M., \& Wisco, B. E. (2017). Negative interpretation bias as a mechanism of the relationship between rejection sensitivity and depressive symptoms. Cognition and Emotion, 31(5), 950-962. doi:http://dx.doi.org/10.1080/02699931.2016. 1185395

Nowland, R., Talbot, R., \& Qualter, P. (2018). Influence of loneliness and rejection sensitivity on threat sensitivity in romantic relationships in young and middle-aged adults. Personality and Individual Differences, 131, 185-190. doi:10.1016/j.paid.2018.04.047

Pachankis, J. (2008). Gay-Related Rejection Sensitivity Scale [Database record]. Retrieved from PsycTESTS. doi:10.1037/t05989-000

Park, L. E. (2007). Appearance-based rejection sensitivity: Implications for mental and physical health, affect, and motivation. Personality and Social Psychology Bulletin, 33, 490-504. doi:10.1177/0146167206296301

Regan, P. (2011). Close relationships. New York: Routledge.

Rohner, R., P., \& Carrasco, M., A. (2014). Teoría de la aceptación-rechazo interpersonal (IPART): Bases conceptuales, método y 
evidencia empírica. Acción Psicológica, 11(2), 9-26. doi:10.5944/ap.11.2.14172

Romero-Canyas, R., Downey, G., Berenson, K., Ayduk, O., \& Kang, J. (2010). Rejection sensitivity and the rejection-hostility link in romantic relationships. Journal of Personality, 78(1), 119-148. doi:10.1111/j.1467-6494.2009.00611.x

Rosales-Sarabia, R., Rivera-Aragón, S., ReidlMartínez, L., \& García-Méndez, M. (2018). Perdón y resentimiento hacia la pareja: Desarrollo y validación de dos escalas. Revista Iberoamericana de Diagnóstico y Evaluación - e Avaliação Psicológica, 2(47), 141-156. doi:10.21865/RIDEP47.2.10.

Rosenbach, C. (2013). Rejection sensitivity: Etiological Aspects and Psychopathological Impact (Doctoral dissertation). Freie Universität Berlin, Germany.

Sato, M., Fonagy, P., \& Luyten, P. (2020). Rejection sensitivity and borderline personality disorder features: The mediating roles of attachment anxiety, need to belong, and self-criticism. Journal of personality disorders, 34(2), 273-288.

doi:10.1521/pedi_2019_33_397

Sang-Hwang, H. (2012). Psychometric properties of the Korean Children's Rejection Sensitivity Questionnaire (K-CRSQ). The Korean Journal of Elementary Counseling. 11(3), 351-369.

Suárez, O. M. (2007). Aplicación del análisis factorial a la investigación de mercados. Caso de estudio. Scientia et technica, 1(35), 281-286. doi:https://doi.org/10.22517/23447214.5443

Sunami, N., Nadzan, M. A., \& Jaremka, L. M. (2019). The bi-dimensional rejection taxonomy: Organizing responses to interpersonal rejection along antisocialprosocial and engaged-disengaged dimensions. Social and Personality Psychology Compass, 13(9), 1-19. doi:https://doi.org/10.1111/spc3.12497

Watson, J., \& Nesdale, D. (2012). Rejection Sensitivity, Social Withdrawal, and Loneliness in Young Adults. Journal of Applied Social Psychology, 42 (8). 19842005. doi:10.1111/j.1559-1816.2012.00927.x

Zhou, J., Li, X., Tian, L., \& Huebner, E. S. (2020). Longitudinal association between low self-esteem and depression in early adolescents: The role of rejection sensitivity and loneliness. Psychology and Psychotherapy: Theory, Research and Practice, 93(1), 54-71.

Zimmer-Gembeck, M., \& Skinner, E. (2015). Adolescent vulnerability and the distress of rejection: Associations of adjustment problems and gender with control, emotions, and coping. Journal of Adolescence, 45, 149159. doi:10.1016/j.adolescence.2015.09.004 


\section{Anexo 1}

Cuestionario de preguntas abiertas sobre sensibilidad al rechazo amoroso

1. ¿Alguna vez, usted se ha sentido rechazado por su pareja?

si su respuesta es afirmativa, por favor explique en qué situaciones.

2. ¿Para usted qué es el rechazo amoroso?

3. ¿Cuándo se siente usted rechazado por su pareja?

4. ¿Qué hace usted cuando su pareja lo rechaza?

5. ¿Qué siente cuando su pareja lo rechaza?

6. ¿A qué se debe que su pareja lo rechace?

\section{Anexo 2}

\section{Escala de Sensibilidad al Rechazo Amoroso (SRA)}

Instrucciones: A continuación se presentan una serie de afirmaciones que describen aspectos de la relación de pareja. Después de leer cada frase, marque con una $(\mathbf{X})$ la opción que mejor describa su situación actual:

\begin{tabular}{|c|c|c|c|c|}
\hline $\begin{array}{c}\text { Totalmente en } \\
\text { desacuerdo }\end{array}$ & En desacuerdo & $\begin{array}{c}\text { Ni de acuerdo ni en } \\
\text { desacuerdo }\end{array}$ & De acuerdo & $\begin{array}{c}\text { Totalmente de } \\
\text { acuerdo }\end{array}$ \\
\hline 1 & 2 & 3 & 4 & 5 \\
\hline
\end{tabular}

\begin{tabular}{|c|c|c|c|c|c|c|}
\hline 1 & Evito hacer cosas por mi pareja porque siento que NO va a corresponderme & 1 & 2 & 3 & 4 & 5 \\
\hline 2 & Pienso que mi pareja me aparta porque le cuesta expresar sus sentimientos & 1 & 2 & 3 & 4 & 5 \\
\hline 3 & Cuando percibo que mi pareja me evita siento culpa & 1 & 2 & 3 & 4 & 5 \\
\hline 4 & Evito etiquetar a mi pareja en redes sociales porque siento que va a ignorarme & 1 & 2 & 3 & 4 & 5 \\
\hline 5 & Pienso que mi pareja se niega a estar conmigo porque hago cosas que le molestan & 1 & 2 & 3 & 4 & 5 \\
\hline 6 & Me siento humillado(a) cuando parece que mi pareja me evita & 1 & 2 & 3 & 4 & 5 \\
\hline 7 & Evito pedirle cosas a mi pareja porque siento que va a negarse & 1 & 2 & 3 & 4 & 5 \\
\hline 8 & Pienso que mi pareja se aparta de mí porque lo desespero & 1 & 2 & 3 & 4 & 5 \\
\hline 9 & Cuando percibo que mi pareja me está evitando siento miedo & 1 & 2 & 3 & 4 & 5 \\
\hline 10 & Evito ser detallista con mi pareja porque siento que va a ignorar mis detalles & 1 & 2 & 3 & 4 & 5 \\
\hline 11 & Pienso que mi pareja me evita porque dejé de cumplir sus expectativas & 1 & 2 & 3 & 4 & 5 \\
\hline 12 & Me siento ansioso(a) cuando noto que mi pareja me está evitando & 1 & 2 & 3 & 4 & 5 \\
\hline 13 & Evito pedirle a mi pareja que salgamos juntos por temor a que se niegue & 1 & 2 & 3 & 4 & 5 \\
\hline 14 & Creo que mi pareja se niega a estar conmigo porque la abrumo con mi cariño & 1 & 2 & 3 & 4 & 5 \\
\hline 15 & Cuando creo que mi pareja se niega a estar conmigo me siento triste & 1 & 2 & 3 & 4 & 5 \\
\hline 16 & Me siento mal cuando considero que mi pareja se niega a estar conmigo & 1 & 2 & 3 & 4 & 5 \\
\hline 17 & Creo que si mi pareja evita escucharme es porque le importo poco & 1 & 2 & 3 & 4 & 5 \\
\hline 18 & Me duele sentir que mi pareja se niega a estar conmigo & 1 & 2 & 3 & 4 & 5 \\
\hline 19 & Evito decirle a mi pareja que la quiero por temor a NO ser correspondido(a) & 1 & 2 & 3 & 4 & 5 \\
\hline 20 & Cuando creo que mi pareja me está evitando me siento inseguro(a) & 1 & 2 & 3 & 4 & 5 \\
\hline 21 & Creo que mi pareja se niega a estar conmigo porque parece fastidiada & 1 & 2 & 3 & 4 & 5 \\
\hline 22 & Evito pedirle a mi pareja que tengamos relaciones porque siento que va a negarse & 1 & 2 & 3 & 4 & 5 \\
\hline 23 & Cuando creo que mi pareja se niega a estar conmigo me siento desesperado(a) & 1 & 2 & 3 & 4 & 5 \\
\hline 24 & Le oculto mis problemas a mi pareja porque siento que se negará a apoyarme & 1 & 2 & 3 & 4 & 5 \\
\hline
\end{tabular}

